\title{
Comparative Analysis of Digital Underwater Video Image Color Balance Correction Algorithms
}

\author{
S. N. Kirillov, P. N. Skonnikov, A. A. Baukov \\ Department of Radio control and Communications, RSREU, Ryazan, Russia \\ E-mail: skonnikovpn@yandex.ru
}

Received: July 08, 2019

\begin{abstract}
Digital video image color balance correction algorithms are analyzed. The procedure of selecting the objects presented in a video sequence is proposed to be used in order to make a comparative analysis of color information: hue and saturation in HSL model, and chroma as well. The indices of color balance correction quality are substantiated. To carry out full-reference assessment, the groups of video sequences were chosen where various underwater vehicles filmed inside and outside the water can be found. The selection of underwater vehicles in images was made; the parameters of color information of the vehicles selected were estimated in the following video images: reference, source (underwater) and processed with the help of color balance correction algorithms under consideration. A comparative analysis of color correction methods for video recordings taken in aquatic environment was carried out in order to identify the best algorithm according to the criterion of the closest color information of objects presented in an underwater video image to color information of corresponding objects not being distorted by aquatic environment. The results of the comparison have shown that the usage of Gray World Assumption algorithm leads to an improvement in hue up to 6.12 times with saturation loss of $8.8 \%$, and the usage of White Patch Retinex algorithm - up to 4.43 times with a loss of saturation of $22.4 \%$.
\end{abstract}

Keywords: video processing, color balance, aquatic environment, object selection, hue, saturation, chroma

\section{INTRODUCTION}

Currently, the work aimed at studying water basin bottom relief, surveying sunken objects, laying and maintaining gas and oil pipelines is actively carried out, scientific research of ocean and sea depths is also conducted. To implement these works, underwater mobile robotic devices are used, generally, with installed digital color video cameras, which make it possible to observe and record scenes at various depths, as well as to control the actions performed.

The quality of underwater video images is significantly influenced by scattering and absorption of light in aquatic environment [1-3]. The degree of light absorption depends on wavelength, as well as on impurities concentration and type. Thus, minimum absorption in the waters of the World Ocean is observed in the range between $470 \ldots 570 \mathrm{~nm}$ [2]. The process of light absorption leads to a reduction in the range of transmitted colors, and, consequently, to distortions in the hue of objects presented in video images taken in an aqueous medium [2]. Distortions of this type significantly reduce the quality of video data, worsen the perception of

(C) S. N. Kirillov, P. N. Skonnikov, A. A. Baukov, 2019 
underwater scene by the operator controlling the device. In addition, these factors make it difficult to detect and recognize the objects located on an oozy bottom. Therefore, to observe underwater scenes with the help of mobile robotic vehicles, an enhanced vision system improving the quality of video image by color balance correction is needed.

At present, various types of corresponding color correction algorithms are known, the results of which are different [4-11], therefore, it seems relevant to perform a comparative analysis of these approaches. The purpose of the analysis is to identify the best algorithm according to the criterion described below for the best approximation of color information of the objects presented in the video image taken in aquatic environment to color information of these objects not distorted by environment.

\section{ALGORITHMS UNDER COMPARISON}

Currently, Bradford's color correction method [4] is known, in which XYZ color space $[12,13]$ of an original image $\left(X_{S}, Y_{S}, Z_{S}\right)$ is linearly converted to the color $\left(X_{D}, Y_{D}, Z_{D}\right)$ of a processed image using the points of equal energy or reference white $\left(X_{W S}, Y_{W S}, Z_{W S}\right)$ and $\left(X_{W D}, Y_{W D}, Z_{W D}\right)$ of source and processed frames, respectively [4]. One of the problems solved in this method is to estimate a reference white point $\left(X_{W S}, Y_{W S}, Z_{W S}\right)$. The solutions to this problem are determined by various color balance correction algorithms based on Bradford method: White Patch Retinex (WPR) [5, 6], Gray World Assumption (GW) [5, 7, 8] and Principal Component Analysis (PCA) [9-11, 14].

The basic idea of WPR algorithm is the assumption that the white area presented in a video scene reflects maximum possible light source for each range [5]. Therefore, in this algorithm, the vector of maximum values of pixel intensities in each channel (R, G and $B$ ) is taken as a reference white point, and the brightest pixels of each range are excluded from consideration.

$\mathrm{GW}$ algorithm assumes that the average color of scene is gray [5]. In accordance with this assumption, a reference white point is calculated by averaging the values of pixel intensities in each range, after excluding the brightest and darkest pixels.

PCA color correction algorithm is based on the principal component method $[9-11,14]$. Colors in RGB space form three-element vectors. In the algorithm considered, at the initial stage, these color vectors are arranged according to the norm of projection onto image average color vector. A reference white point represents a vector of dominant direction in the set of selected color vectors, which is calculated using the principal component analysis method [14].

\section{EXPERIMENT DESCRIPTION}

\section{General principles of comparison}

The goal of applying color balance correction algorithms in enhanced vision systems for underwater mobile robotic vehicles is to bring the colors of objects presented in the frame closer to more "natural" ones. Here, "natural" colors are the colors not being distorted by aquatic environment. To assess the quality of color balance correction in order to reduce the influence of aquatic environment, it is appropriate to use reference color parameters of objects of interest, where color parameters of these objects are used as a reference under normal conditions, that is, in daylight out of water. 
For the experiment, the groups of videos containing four different underwater vehicles were chosen [15-17]. Three of them, having a bright yellow color, were presented in video recordings taken both underwater and out of water. The fourth apparatus is a priori known to have white color; therefore, in this group, only video records obtained in aquatic environment are used. In the video recordings selected, objects move and change their spatial orientation as the result of which underwater vehicles and their individual elements have different lighting. In addition, scenes with different background color are used for underwater video recordings.

To exclude background and extraneous objects from the analysis, an automated selection of the object of interest is performed. Then for the selected object the average values of color parameters are calculated. In this case, averaging is performed over the pixels of the object selected and over the frames. Further, these parameters calculated for reference, source and processed video are used to compare color balance correction algorithms.

Hue, saturation and chroma have been chosen as the analyzed color parameters. Hue and saturation are used when presenting colors in HSI (HSV) and HSL models [18-20]. However, despite widespread use of these color spaces, the use of saturation as a measure of colorfulness can lead to incorrect results in dark areas of an image. Therefore, for additional consideration of colorfulness, chroma values of the object selected are also calculated.

\section{Comparison procedure}

When analyzing the quality of color balance correction, images representing the frames of the chosen videos are used: reference image $I_{\text {ref }}$, original image $I_{\text {source }}$ to be corrected for color balance, and the results of processing using PCA $I_{P C A}$, WPR $I_{W P R}$, GW $I_{G W}$ algorithms. The image data is initially presented as a component of red, green, and blue $I=\{R, G, B\}$. The color of the object of interest is calculated in the same way for all images $I_{\text {ref }}, I_{\text {source }}, I_{P C A}, I_{W P R}, I_{G W}$.

On the first stage of a comparison procedure of the algorithms indicated, the image is transferred to HSL color space. For each pixel $i=\{r, g, b\}$ of image $I$, a transformation is performed, as a result of which pixel $i$ is represented as components of hue, saturation and lightness $i=\{h, s, l\}$, respectively [18, 19]. In addition, chroma values are calculated [18, 19]:

$$
c=\max (r, g, b)-\min (r, g, b) .
$$

On the second stage, the object of interest is selected, that is, a mask $M$ is formed, $m$ pixels of which are defined as follows:

$$
\left\{\begin{array}{l}
m=1, \quad \text { if }\left\{\begin{array}{l}
h \in\left[H_{\min }, H_{\max }\right] ; \\
s \in\left[S_{\min }, S_{\max }\right] ; \\
l \in\left[L_{\min }, L_{\max }\right] ;
\end{array}\right. \\
m=0, \quad \text { otherwise. }
\end{array}\right.
$$

where $H_{\min }, H_{\max }, S_{\min }, S_{\max }, L_{\min }, L_{\max }$ are lower and upper thresholds of the channels of hue $H$, saturation $S$ and lightness $L$, chosen experimentally for each video record. In order not to take into account excessively illuminated or darkened areas of the object, where values $h$ and $s$ may turn out to be incorrect, lightness thresholds were chosen not equal to the limits of dynamic range of channel $L$, i.e. $L_{\min }>0, L_{\max }<1$. 
In Fig. 1 frames $I_{\text {ref }}$ of video recordings of various scenes and selected objects for clarity being presented in the form of element wise multiplication result $I_{\text {ref }} \cdot M$ are shown.
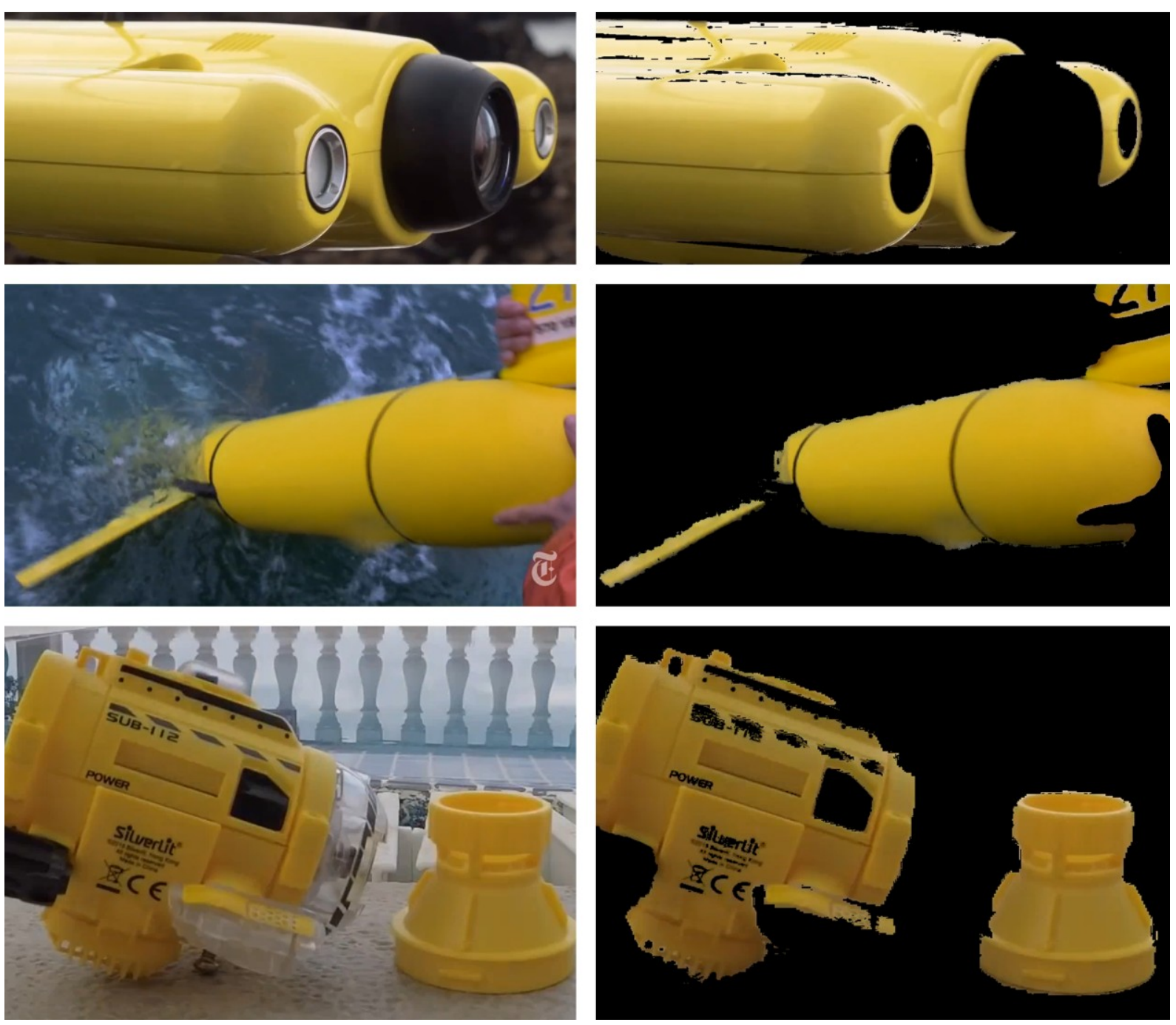

Figure 1. Frames of source video recordings of various scenes (on the left) and the results of objects selection (on the right)

On the third stage, color parameters of the objects selected are averaged by pixels and frames. The average hue value of the selected object is determined by the formula

$$
\bar{H}=\sum_{N_{f}} \sum_{N_{p}} h \cdot m / \sum_{N_{f}} \sum_{N_{p}} m
$$

where $N_{p}$ is the total number of pixels in a frame, $N_{f}$ is the number of frames in a video. For other color parameters, the calculation is performed in the same way. This operation is performed for all video recordings: reference, source and processed by PCA, WPR, GW algorithms. Thus, at this stage, the parameters of average hue $\bar{H}_{\text {ref }}, \bar{H}_{\text {source }}, \bar{H}_{P C A}, \bar{H}_{W P R}, \bar{H}_{G W}$, average saturation $\bar{S}_{\text {ref }}, \bar{S}_{\text {source }}, \bar{S}_{P C A}, \bar{S}_{W P R}, \bar{S}_{G W}$ and average chroma $\bar{C}_{r e f}, \bar{C}_{\text {source }}, \bar{C}_{P C A}$, $\bar{C}_{W P R}, \bar{C}_{G W}$ are determined. 
Fig. 2 as an example shows the frame of an original video and the results of the image processed by the algorithms considered. This image shows only the selected objects, since only the pixels of these objects are taken into account when analyzing the algorithms.

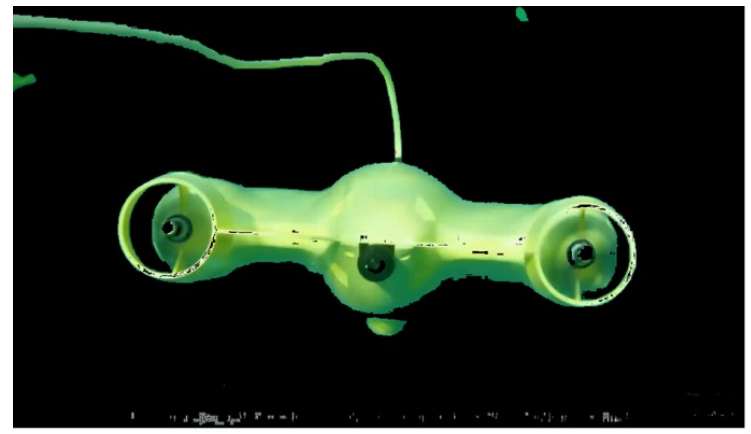

(a)

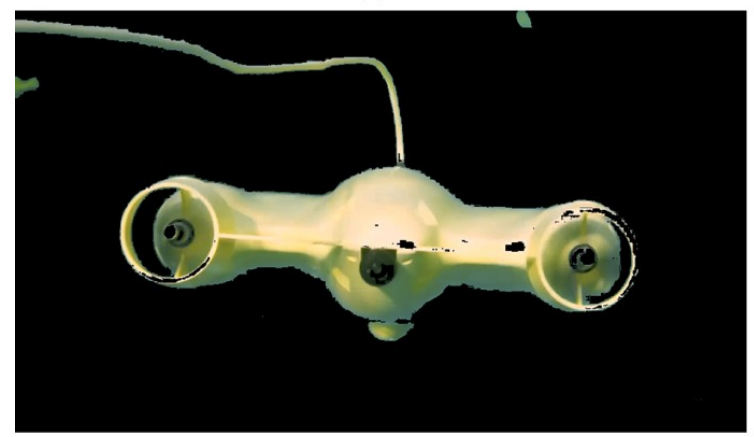

(c)

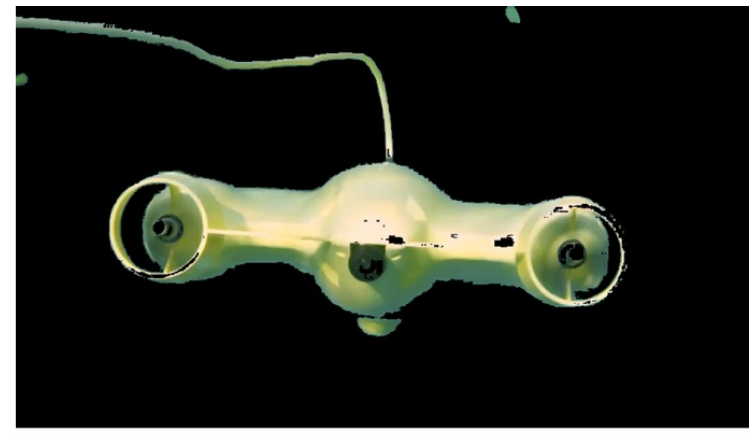

(b)

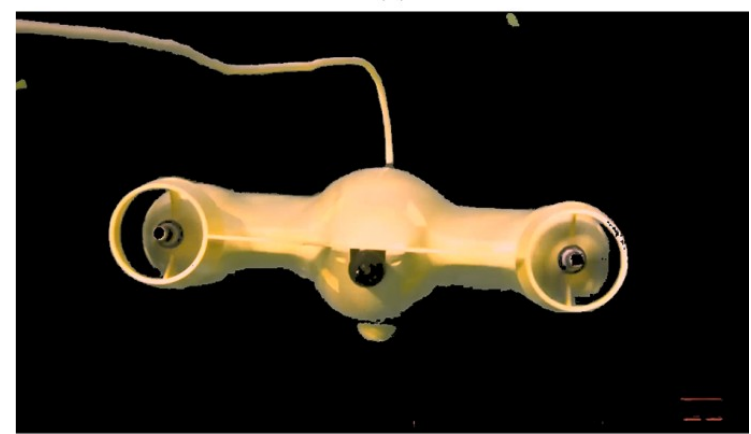

(d)

Figure 2. The selected object on the source image (a) and on the images processed using PCA (b), WPR (c), and GW (d) algorithms

The values of color averaged parameters of the object in this frame (at $N_{f}=1$ ) were: $\bar{H}_{\text {source }}=0.31, \quad \bar{H}_{P C A}=0.24, \quad \bar{H}_{W P R}=0.21, \quad \bar{H}_{G W}=0.11, \quad \bar{S}_{\text {source }}=0.56, \quad \bar{S}_{P C A}=0.4$, $\bar{S}_{W P R}=0.43, \bar{S}_{G W}=0.62$.

For the scenes with yellow objects, parameters $\bar{H}, \bar{S}$ and $\bar{C}$ themselves are not indicators of improved color balance. The difference $\Delta \bar{H}=\bar{H}-\bar{H}_{r e f}$ is used as a measure of hue proximity to reference values. Here $\bar{H}$ is seen as one of the values $\bar{H}_{\text {source }}, \bar{H}_{P C A}, \bar{H}_{W P R}$, $\bar{H}_{G W}$. The measure of video image hue improvement due to the application of an algorithm is determined as a ratio $G_{H}=\Delta \bar{H}_{\text {source }} / \Delta \bar{H}$ by the criterion of approaching a reference value. Saturation and chroma gain are calculated as ratios $G_{S}=\bar{S} / \bar{S}_{r e f}, G_{C}=\bar{C} / \bar{C}_{r e f}$, respectively. Improvement in color balance correction quality corresponds to the increase in value $\left|G_{H}\right|$ and the approximation of values $G_{S}$ and $G_{C}$ - to a unity.

A group of videos containing a white underwater vehicle was analyzed in a slightly different way. Firstly, hue values were not taken into account, since this parameter does not make sense for a white object. Secondly, since for a white object $\bar{S}_{r e f}=0$ and $\bar{H}_{\text {ref }}=0$, an indicator showing the quality of color balance correction algorithms application are the coefficients of decreasing saturation $R_{S}=\bar{S}_{\text {source }} / \bar{S}$ and chroma $R_{C}=\bar{C}_{\text {source }} / \bar{C}$. Accordingly, in this case the larger the values of $R_{S}$ and $R_{C}$, the better the algorithm for color balance correction is. 


\section{EXPERIMENTAL RESULTS}

Table 1 shows mean values of hue $\bar{H}$, saturation $\bar{S}$ and chroma $\bar{C}$ of selected objects, as well as coefficients $G_{H}, G_{S}, G_{C}$ calculated on their basis.

Table 1. Averaged color parameters of selected objects and indicators of color balance improvement for scenes with yellow objects

\begin{tabular}{|c|l|c|c|c|c|c|c|}
\hline Scene & \multicolumn{1}{|c|}{ Video } & $\overline{\boldsymbol{H}}$ & $\overline{\boldsymbol{S}}$ & $\overline{\boldsymbol{C}}$ & $\boldsymbol{G}_{\boldsymbol{H}}$ & $\boldsymbol{G}_{\boldsymbol{S}}$ & $\boldsymbol{G}_{\boldsymbol{C}}$ \\
\hline \multirow{6}{*}{1} & Reference & 0.125 & 0.584 & 85 & - & 1 & 1 \\
\cline { 2 - 8 } & Original & 0.308 & 0.576 & 82 & 1 & 0.987 & 0.9639 \\
\cline { 2 - 8 } & PCA & 0.263 & 0.345 & 48.8 & 1.33 & 0.592 & 0.574 \\
\cline { 2 - 8 } & WPR & 0.254 & 0.339 & 41.3 & 1.42 & 0.58 & 0.486 \\
\cline { 2 - 8 } & GW & 0.095 & 0.754 & 91 & -6.12 & 1.291 & 1.07 \\
\hline \multirow{3}{*}{2} & Reference & 0.109 & 0.633 & 118.1 & - & 1 & 1 \\
\cline { 2 - 8 } & Original & 0.259 & 0.283 & 56 & 1 & 0.448 & 0.475 \\
\cline { 2 - 8 } & PCA & 0.237 & 0.267 & 55.3 & 1.14 & 0.42 & 0.468 \\
\cline { 2 - 8 } & WPR & 0.236 & 0.267 & 55.3 & 1.14 & 0.42 & 0.468 \\
\cline { 2 - 8 } & GW & 0.236 & 0.267 & 55.3 & 1.14 & 0.42 & 0.468 \\
\hline \multirow{3}{*}{3} & Reference & 0.128 & 0.765 & 147 & - & 1 & 1 \\
\cline { 2 - 8 } & Original & 0.424 & 0.756 & 101.6 & 1 & 0.989 & 0.69 \\
\cline { 2 - 8 } & PCA & 0.199 & 0.57 & 87.7 & 4.2 & 0.746 & 0.596 \\
\cline { 2 - 7 } & WPR & 0.195 & 0.594 & 89.3 & 4.43 & 0.776 & 0.607 \\
\cline { 2 - 8 } & GW & 0.177 & 0.698 & 108.7 & 6.12 & 0.912 & 0.739 \\
\hline
\end{tabular}

Analysis of this table shows that all color balance correction algorithms considered improve the hue $\left(\left|G_{H}\right|>1\right)$, but hue colorfulness is distorted. In this case, PCA and WPR algorithms reduce saturation and chroma, and GW algorithm provides the least distortion of these parameters. However, when using this algorithm, the object as a result of processing may be oversaturated $\left(G_{S}>1, G_{C}>1\right)$. The value $G_{H}<-1$ for $\mathrm{GW}$ algorithm shows that an overshoot occurs, i.e., as a result of processing the hue approaches the reference, but the hue change is larger than necessary: $\left|\Delta \bar{H}_{\text {source }}\right|<\left|\bar{H}_{\text {source }}-\bar{H}_{G W}\right|<\left|2 \Delta \bar{H}_{\text {source }}\right|$.

Values of $\bar{S}, \bar{C}$ parameters and $R_{S}, R_{C}$ quality indicators for a white object are given in Table 2.

Table 2. Averaged parameters of colorfulness of selected objects and indicators of its reduction for scenes with white objects

\begin{tabular}{|c|c|c|c|c|}
\hline Video & $\overline{\boldsymbol{S}}$ & $\overline{\boldsymbol{C}}$ & $\boldsymbol{R}_{\boldsymbol{S}}$ & $\boldsymbol{R}_{\boldsymbol{C}}$ \\
\hline Original & 0.259 & 42.2 & 1 & 1 \\
\hline PCA & 0.211 & 31.3 & 1.23 & 1.35 \\
\hline WPR & 0.194 & 28.7 & 1.33 & 1.47 \\
\hline GW & 0.977 & 113.1 & 0.27 & 0.37 \\
\hline
\end{tabular}

From the analysis of Table 2 it can be seen that PCA and WPR algorithms reduce saturation and chroma of an object having a white color, that is, improve the image. GW algorithm, in contrast, leads to a false "coloring" of an object. 


\section{CONCLUSION}

The results of color parameter analysis of the underwater objects highlighted in video have shown that GW algorithm provides the best approximation to the standard. However, this algorithm in some cases leads to the overshoot of all considered parameters and false staining of less saturated objects. The improvement in hue as a result of applying GW algorithm reaches 6.12 times with a saturation loss of $8.8 \%$.

For tasks in which the overshoot of color parameters and false staining are not allowed, among the considered color balance correction algorithms, WPR algorithm has the best quality indicators. The improvement in hue in this case reaches 4.43 times with the loss in saturation of $22.4 \%$.

\section{REFERENCES}

1. Dolin, L. S., \& Levin, I. M. (1991). Spravochnik po teorii podvodnogo videniya [Handbook of underwater vision theory]. Leningrad: Gidrometeoizdat (in Russian).

2. Fisenko, T. Yu., \& Fisenko, V. T. (2012). Issledovanie i razrabotka metodov uluchsheniya podvodnykh izobrazheniy [Research and development of improving underwater images methods]. In: Sbornik trudov X Mezhdunarodnoy konferentsii "Prikladnaya optika - 2012” [Proceedings of the X International Conference “Applied Optics - 2012”] (pp. 294 - 298). (in Russian).

3. Kirillov, S. N., \& Kostkin, I. V. (2016). Besprovodnaya sistema vysokoskorostnoy podvodnoy opticheskoy svyazi dlya peredachi videodannykh s borta podvodnogo apparata v rezhime real'nogo vremeni [Wireless system of high-speed underwater optical communication for real time transmission of video data]. Radiotekhnika [Radioengineering], 11, 80-89 (in Russian).

4. Lindbloom, B. Chromatic adaptation. Retrieved from http://www.brucelindbloom.com/index.html?Eqn_ ChromAdapt.html.

5. Ebner, M. (2007). Color constancy. Chichester, West Sussex: John Wiley \& Sons.

6. Cardei, V. C., \& Funt, B. (1999). Committee-based color constancy. In: Proceedings of the IS\&T/SID Seventh Color Imaging Conference: Color Science, Systems and Applications (pp. 311-313).

7. Buchsbaum, G. (1980). A spatial processor model for object colour perception. Journal of the Franklin Institute, 310(1), 337-350.

8. Judd, D. B. (1960). Appraisal of Land's work on two-primary color projections. Journal of the Optical Society of America, 50(3), 254-268.

9. Hotelling, H. (1933). Analysis of a complex of statistical variables into principal components. Journal of Educational Psychology, 24, 417-441, and 498-520.

10. Jolliffe, I. T. (2002). Principal component analysis. Series: Springer Series in Statistics, 2nd ed., Springer, NY, XXIX.

11. Cheng, D., Prasad, D. K., \& Brown, M. S. (2014). Illuminant estimation for color constancy: Why spatialdomain methods work and the role of the color distribution. Journal of the Optical Society of America A, 5, 1049-1058.

12. Gonzalez, R. C., \& Woods, R. E. (2012). Tsifrovaya obrabotka izobrazheniy [Digital Image Processing]. Moscow: Tekhnosfera (in Russian).

13. Smith, T., \& Guild, J. (1931-32). The C.I.E. colorimetric standards and their use. Transactions of the Optical Society, 33(3), 73-134. doi:10.1088/1475-4878/33/3/301.

14. Abdi, H., \& Williams, L.J. (2010). Principal component analysis. Wiley Interdisciplinary Reviews: Computational Statistics, 2(4), 433-459. doi:10.1002/wics.101.

15. Silverlit remote control submarine Spycam Aqua running video. Retrieved from https://www.youtube.com/ watch?v=eiB2iviEq2Q.

16. 5 AMAZING Underwater Drones. Retrieved from https://www.youtube.com/watch?v=KekgdyGACdY. 
"Instrumentation Engineering, Electronics and Telecommunications - 2019" Proceedings of the V International Forum (Izhevsk, Russia, November 20-22, 2019)

17. Herkewitz, W. Ocean Drones Plumb New Depths. Retrieved from https://www.nytimes.com/2013/11/12/ science/earth/ocean-drones-plumb-new-depths.html?smid=yt-nytimes.

18. Oleari, C. (2016). Standard colorimetry: definitions, algorithms and software. Chichester, West Sussex: John Wiley \& Sons.

19. Malacara, D. (2011). Color vision and colorimetry: theory and applications. 2nd ed. Bellingham, Washington USA: Society of Photo-Optical Instrumentation Engineers (SPIE) Press.

20. Gonsales, R. C., Woods, R. E., \& Eddins, S. L. (2006). Tsifrovaya obrabotka izobrazhenij v srede $M A T L A B$ [Digital image processing using MATLAB]. Moscow: Tekhnosfera (in Russian). 